\title{
Sulfonamide Derivatives of 2-Amino-1-phenylethane as Suitable Cholinesterase Inhibitors
}

\author{
Muhammad A Abbasi ${ }^{1 *}$, Sagheer Ahmad1', Aziz-ur-Rehman', Shahid Rasool', \\ Khalid M Khan ${ }^{2}$, Muhammad Ashraf ${ }^{3}$, Rumana Nasar ${ }^{3}$ and Tayaba Ismail ${ }^{3}$ \\ ${ }^{1}$ Department of Chemistry, Government College University, Lahore-54000, ${ }^{2}$ HEJ Research Institute of Chemistry, International \\ Center for Chemical and Biological Sciences, University of Karachi, Karachi-75270, ${ }^{3}$ Department of Biochemistry and \\ Biotechnology; The Islamia University of Bahawalpur, Bahawalpur-63100, Pakistan.
}

*For correspondence: Email: atrabbasi@yahoo.com, abbasi@gcu.edu.pk; Tel: +92-42-111000010 ext 266

Received: 6 September 2013

Revised accepted: 19 March 2014

\begin{abstract}
Purpose: To evaluate the enzyme inhibition activity of $N$-substituted sulfamoyl derivatives of 2-amino-1phenylethane as probable new drug candidates for the treatment of Alzheimer's diseases.

Methods: A series of sulfamoyl derivatives, 3a-l, of 1-amino-2-phenylethane (1) were synthesized by reacting with various aryl sulfonyl chlorides, 2a-l, in the presence of aqueous $\mathrm{Na}_{2} \mathrm{CO}_{3}$ solution under definite $\mathrm{pH}$ control. All the synthesized molecules were screened against three enzymes, acetyl cholinesterase (AChE), butyryl cholinesterase (BChE) and lipoxygenase (LOX). The synthesized derivatives were further characterized by infra-red spectroscopy (IR), nuclear magnetic resonance $\left(^{1} \mathrm{H}\right.$ $N M R$ ) and electron ionization-mass spectrometry (EI-MS) for structure elucidation.

Results: Screening against acetyl cholinesterase (AChE), butyryl cholinesterase (BChE) and lipoxygenase (LOX) showed these molecules to be suitable inhibitors of cholinesterase enzymes, AChE and $B C h E$, relative to eserine, the reference standard. The molecule, $3 c$, remained effective with $50 \%$ inhibitory concentration $\left(I C_{50}\right)$ value of $82.93 \pm 0.15 \mu \mathrm{M}$ (relative to eserine with $I C_{50}$ value of $0.04 \pm$ $0.0001 \mu \mathrm{M}$ ) against $A C h E ;$ similarly $3 d$ was active against $B C h E$ with $I C_{50}$ value of $45.65 \pm 0.48 \mu \mathrm{M}$ compared to eserine with $I C_{50}$ value of $0.85 \pm 0.00 \mu \mathrm{M}$. The molecule, $3 f$, was inactive against all the three enzymes.

Conclusion: Overall, the results indicate that these compounds are active against cholinesterase enzymes but less potent against lipoxygenase enzyme.
\end{abstract}

Keywords: 1-Amino-2-phenylethane, Aryl sulfonyl chlorides, Cholinesterase enzymes, Lipoxygenase

Tropical Journal of Pharmaceutical Research is indexed by Science Citation Index (SciSearch), Scopus, International Pharmaceutical Abstract, Chemical Abstracts, Embase, Index Copernicus, EBSCO, African Index Medicus, JournalSeek, Journal Citation Reports/Science Edition, Directory of Open Access Journals (DOAJ), African Journal Online, Bioline International, Open-J-Gate and Pharmacy Abstracts

\section{INTRODUCTION}

Sulfonamides were the first antibacterial agents used successfully for the treatment of infectious diseases in human beings. These were also employed for the treatment of various infections [1]; as antimicrobial, antithyroid, antitumor and antimalarial agents [2]; as inhibitor of carbonic anhydrase [2]; for the treatment of diabetes [3], HIVIAIDS [4] and bacterial infections in the animals [5]. Sulfonamides inhibit the formation of folic acid necessary for bacterial growth by competing with $p$-aminobenzoic acid for dihydropteroate synthase enzyme and ultimately inhibit the synthesis of purine and DNA [6].

Acetyl cholinesterase (AChE, EC 3.1.1.7) and butyryl cholinesterase (BChE, EC 3.1.1.8) belong to a group of enzymes including serine hydrolases and are key components of cholinergic brain synapses and neuromuscular 
junctions. These catalyze hydrolysis of neurotransmitter acetylcholine and terminate nerve impulse in cholinergic synapses [7]. BChE is present significantly in Alzheimer's plaques than the normal age related non dementia of brains. The cholinesterase inhibitors increase the amount of acetylcholine, for neuronal and neuromuscular transmission, reversibly or irreversibly [8].

The seeking of new cholinesterase and lipoxygenase enzyme inhibitors is thought to be an important strategy to inaugurate new drug candidates for the treatment of Alzheimer's disease and other related ones. The previous work by our group [9-12] has revealed that different structural changes in the molecule by substitution have a great influence on the biological activities. The objective of this work was to synthesize less toxic and more efficient sulfonamides against AChE, BChE and LOX enzymes, derived from 1-amino-2-phenylethane.

\section{EXPERIMENTAL}

\section{Materials and instruments}

Melting points of synthesized compounds were recorded with the help of Griffin and George melting apparatus. Purity of synthesized molecules was checked by thin layer chromatography (TLC) on G-25-UV plates coated with silica gel using ethyl acetate and $n$ hexane as solvent system. Detection wavelength was $254 \mathrm{~nm}$ by using ceric sulphate reagent. The IR spectra were recorded in $\mathrm{KBr}$ pellet method by using Jasco 320-A spectrophotometer with wave number taken in $\mathrm{cm}^{-1} . \mathrm{CH}_{3} \mathrm{OD}$ was used to record ${ }^{1} \mathrm{H}-\mathrm{NMR}$ spectra on Bruker spectrometer working at $300 \mathrm{MHz}$. Mass spectra El-MS were recorded with the help of JMS-HX-110 spectrometer in Finnigan MAT-112 instrument. 1Amino-2-pheylethane and substituted aryl sulfonyl chlorides were purchased from Merck and Alfa Aesar through local suppliers. The solvents employed in synthesis were of analytical grade.

\section{General procedure for the synthesis of different sulfonamides (3a-l)}

1-Amino-2-phenylethane $(1 ; 0.1 \mathrm{mmol})$ was suspended in $100 \mathrm{~mL}$ distilled water in a $250 \mathrm{~mL}$ round bottom flask and $\mathrm{pH}$ was kept strictly 9-10 by the addition of $10 \%$ aqueous solution of $\mathrm{Na}_{2} \mathrm{CO}_{3}$. The various aryl sulfonyl chlorides $(2 \mathrm{a}-\mathrm{I})$ were added to the flask and the decrease in $\mathrm{pH}$ was avoided by the again addition of aq. $\mathrm{Na}_{2} \mathrm{CO}_{3}$ solution. The reaction mixture was stirred for 3-4 hours 4-5 hours and monitored with TLC ( $n$ hexane:EtOAc, 70:30) till the completion of reaction by single spot on TLC plate. After

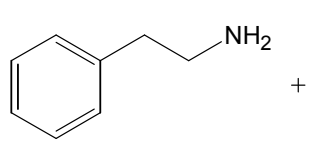

1<smiles>[R]S(=O)(=O)Cl</smiles>

2a-1

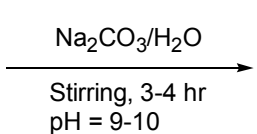

$\mathrm{pH}=9-10$

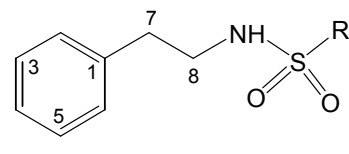

3a-1

\begin{tabular}{|c|c|c|c|c|c|}
\hline Compd. & $\mathbf{R}$ & Compd. & $\mathbf{R}$ & Compd. & $\mathbf{R}$ \\
\hline $3 a$ & & $3 e$ & & $3 \mathbf{i}$ & \\
\hline $3 b$ & & $3 f$ & & $3 \mathbf{j}$ & \\
\hline $3 c$ & & $3 g$ & & $3 k$ & \\
\hline $3 d$ & & $3 h$ & & 31 & \\
\hline
\end{tabular}


confirmation by single spot, $3 \mathrm{~mL}$ of dilute $\mathrm{HCl}$ was added to adjust the $\mathrm{pH}$ of the reaction mixture to 3 - 4 . The synthesized compounds were collected by filtration and washed with distilled water. The re-crystallization was carried out by methanol.

\section{Enzyme inhibition assays}

\section{Cholinesterase assay}

The AChE and BChE inhibition activity were carried out according to the method reported in the literature [13] with minor changes. Volume of the reaction mixture was $100 \mu \mathrm{L}$ containing $60 \mu \mathrm{L}$ $\mathrm{Na}_{2} \mathrm{HPO}_{4}$ buffer (50 mM, pH 7.7), $10 \mu \mathrm{L}$ test compound (0.5 mM well $\left.{ }^{-1}\right)$ and $10 \mu \mathrm{L}$ (0.5 unit well $^{-1} \mathrm{BChE}$ or 0.005 unit well ${ }^{-1} \mathrm{AChE}$ ) enzyme. The contents were mixed, pre-read at $405 \mathrm{~nm}$ and pre-incubated for $10 \mathrm{~min}$ at $37^{\circ} \mathrm{C}$. The reaction was started by the addition of $10 \mu \mathrm{L}(0.5$ $\mathrm{mM}$ well $^{-1}$ ) substrate (acetylthiocholine iodide for AChE and butyrylthiocholine chloride for BChE) and $10 \mu \mathrm{L}$ DTNB $\left(0.5 \mathrm{mM}\right.$ well $\left.{ }^{-1}\right)$. After $15 \mathrm{~min}$ of incubation at $37^{\circ} \mathrm{C}$, absorbance was measured at $405 \mathrm{~nm}$ using 96-well plate reader Synergy HT, Biotek, USA. All experiments were carried out with their respective controls in triplicate. Eserine $\left(0.5 \mathrm{mM}^{-1}\right.$ well $\left.{ }^{-1}\right)$ was used as a positive control. Inhibition was calculated using Eq 1.

Inhibition $(\%)=\{(\mathrm{Ac}-\mathrm{At}) / \mathrm{Ac}\} 100$

where $\mathrm{Ac}=$ absorbance of control and At $=$ absorbance of test compound.

$\mathrm{IC}_{50}$ values were calculated using EZ-Fit Enzyme Kinetics software (Perrella Scientific Inc. Amherst, USA). IC $\mathrm{C}_{50}$ values were calculated from the graph by a serial dilution of compounds to different concentrations. These are mean of three independent experiments.

\section{Lipoxygenase assay}

Lipoxygenase (LOX) activity was assayed according to the method of Baylac \& Racine [14] with slight modifications. Total volume of lipoxygenase assay mixture was $200 \mu \mathrm{L}$ containing $150 \mu \mathrm{L} \mathrm{Na} \mathrm{PO}_{4}$ buffer $(100 \mathrm{mM} \& \mathrm{pH}$ 8.0), $10 \mu \mathrm{L}$ test compound $\left(0.5 \mathrm{mM}\right.$ well $\left.{ }^{-1}\right)$ and 15 $\mu \mathrm{L}\left(600\right.$ units well $\left.^{-1}\right)$ enzyme. The contents were mixed, pre-read at $234 \mathrm{~nm}$ and pre-incubated for 10 minutes at $25^{\circ} \mathrm{C}$. The reaction was initiated by addition of $25 \mu \mathrm{L}$ substrate solution. The change in absorbance was observed after $6 \mathrm{~min}$ at $234 \mathrm{~nm}$ using 96-well plate reader Synergy $\mathrm{HT}$, Biotek, USA. All reactions were performed in triplicates. The positive and negative controls were included in the assay. Baicalein $(0.5 \mathrm{mM}$ well $^{-1}$ ) was used as a positive control. The percentage inhibition (\%) and $\mathrm{IC}_{50}$ values were calculated by the same method as described for cholinesterase enzymes.

\section{Statistical analysis}

All the measurements were carried out in triplicate and statistical analysis was performed by Microsoft Excel 2010. The results are presented as mean \pm SEM with $90 \% \mathrm{CL}$.

\section{RESULTS}

\section{Chemistry}

The molecules, 3a-I, were synthesized by coupling 1-amino-2-phenylethane (1) with different aryl sulfonyl chlorides (2a-I) in a weak basic aqueous media with dynamic $\mathrm{pH}$ control. The products were yielded after stirring of 4-5 hours and isolated by filtration after acidifying up to $\mathrm{pH}$ of 4-5. Acidic $\mathrm{pH}$ is necessary for good yield of the products but high acidity has negative effect. The structural analysis was performed through spectral data.

\section{Spectral characterization of the synthesized molecules}

\section{(3a-I)N-(2-Phenylethyl)-4-methylbenzene sulfonamide (3a)}

White crystalline solid; Yield: $94.72 \%$; m.p.: 98 ${ }^{\circ} \mathrm{C}$; Mol. formula: $\mathrm{C}_{15} \mathrm{H}_{17} \mathrm{NO}_{2} \mathrm{~S}$; Mol. mass: 275 $\mathrm{gmol}^{-1}$; IR $\left(\mathrm{KBr}, \mathrm{cm}^{-1}\right) v_{\text {max }}: 3310$ (N-H stretching), 2930 (C-H stretching of aromatic ring), 2740 ($\mathrm{CH}_{2}$ stretching), $1601 \quad(\mathrm{C}=\mathrm{C}$ stretching of aromatic ring), 1320 ( $\mathrm{S}=\mathrm{O}$ stretching); ${ }^{1} \mathrm{H}-\mathrm{NMR}$ (300 MHz, CD3OD): $\delta / p p m ~ 7.68(d, J=8.1 \mathrm{~Hz}$, $2 \mathrm{H}, \mathrm{H}-2$ ', H-6'), 7.33 (d, J = 8.1 Hz, 2H, H-3', H$\left.5^{\prime}\right), 7.23$ (dd, J = 7.5, $\left.1.5 \mathrm{~Hz}, 2 \mathrm{H}, \mathrm{H}-2, \mathrm{H}-6\right), 7.15$ $(\mathrm{t}, J=7.8 \mathrm{~Hz}, 1 \mathrm{H}, \mathrm{H}-4), 7.09$ (dd, $J=7.8,1.2 \mathrm{~Hz}$, $2 \mathrm{H}, \mathrm{H}-3, \mathrm{H}-5), 3.03(\mathrm{t}, \mathrm{J}=7.8 \mathrm{~Hz}, 2 \mathrm{H}, \mathrm{H}-8), 2.69$ (t, J = $7.2 \mathrm{~Hz}, 2 \mathrm{H}, \mathrm{H}-7), 2.40\left(\mathrm{~s}, 3 \mathrm{H}, \mathrm{CH}_{3}-4\right)$; EIMS: $(\mathrm{m} / \mathrm{z}): 275[\mathrm{M}]^{+}, 155\left[\mathrm{C}_{7} \mathrm{H}_{7} \mathrm{SO}_{2}\right]^{+}, 120$ $\left[\mathrm{C}_{8} \mathrm{H}_{10} \mathrm{~N}\right]^{+}, 105\left[\mathrm{C}_{8} \mathrm{H}_{9}\right]^{+}, 91\left[\mathrm{C}_{7} \mathrm{H}_{7}\right]^{+}, 77\left[\mathrm{C}_{6} \mathrm{H}_{5}\right]^{+}, 65$ $\left[\mathrm{C}_{5} \mathrm{H}_{5}\right]^{+}, 51\left[\mathrm{C}_{4} \mathrm{H}_{3}\right]^{+}$.

\section{N-(2-Phenylethyl)-4-(1,1,1-trimethyl)methyl benzenesulfonamide (3b)}

Brownish black crystalline solid; Yield: $94.18 \%$; m.p.: $112{ }^{\circ} \mathrm{C}$; Mol. formula: $\mathrm{C}_{18} \mathrm{H}_{23} \mathrm{NO}_{2} \mathrm{~S}$; Mol. mass: $317 \mathrm{gmol}^{-1}$; IR $\left(\mathrm{KBr}, \mathrm{cm}^{-1}\right) V_{\max }: 3301(\mathrm{~N}-\mathrm{H}$ stretching), 2927 (C-H stretching of aromatic ring), $2701 \quad\left(-\mathrm{CH}_{2} \quad\right.$ stretching $), \quad 1617 \quad(\mathrm{C}=\mathrm{C}$ stretching of aromatic ring), $1301 \quad(\mathrm{~S}=\mathrm{O}$ stretching); ${ }^{1} \mathrm{H}-\mathrm{NMR}(300 \mathrm{MHz}, \mathrm{CD} 3 \mathrm{OD}): \delta / \mathrm{ppm}$ $7.73\left(\mathrm{~d}, J=8.4 \mathrm{~Hz}, 2 \mathrm{H}, \mathrm{H}-2^{\prime}, \mathrm{H}-6^{\prime}\right), 7.61$ (d, $J=$ $\left.8.4 \mathrm{~Hz}, \mathrm{H}-3^{\prime}, \mathrm{H}-5^{\prime}\right), 7.23$ (d, J = 7.5 Hz, 2H, H-2, $\mathrm{H}-6), 7.16(\mathrm{t}, J=7.5 \mathrm{~Hz}, 1 \mathrm{H}, \mathrm{H}-4), 7.09$ (d, $J=6.9$ $\mathrm{Hz}, \mathrm{H}-3, \mathrm{H}-5), 3.05$ (t, $J=7.2 \mathrm{~Hz}, \mathrm{H}-8), 2.70$ (t, $J$ 
$=7.8 \mathrm{~Hz}, \mathrm{H}-7), 1.34\left(\mathrm{~s}, 9 \mathrm{H},\left(\mathrm{CH}_{3}\right)_{3} \mathrm{C}-4\right.$ '); EIMS $(\mathrm{m} / \mathrm{z}): 317[\mathrm{M}]^{+}, 197\left[\mathrm{C}_{10} \mathrm{H}_{13} \mathrm{SO}_{2}\right]^{+}, 133\left[\mathrm{C}_{10} \mathrm{H}_{13}\right]^{+}$, $120\left[\mathrm{C}_{8} \mathrm{H}_{10} \mathrm{~N}\right]^{+}, \quad 105\left[\mathrm{C}_{8} \mathrm{H}_{9}\right]^{+}, \quad 91\left[\mathrm{C}_{7} \mathrm{H}_{7}\right]^{+}, \quad 77$ $\left[\mathrm{C}_{6} \mathrm{H}_{5}\right]^{+}, 65\left[\mathrm{C}_{5} \mathrm{H}_{5}\right]^{+}, 51\left[\mathrm{C}_{4} \mathrm{H}_{3}\right]^{+}$.

\section{N-(2-Phenylethyl)-2,4,6-trimethylbenzene sulfonamide (3c)}

Brownish black viscous liquid; Yield: 94.77\%; Mol. formula: $\mathrm{C}_{17} \mathrm{H}_{21} \mathrm{NO}_{2} \mathrm{~S}$; Mol. mass: $303 \mathrm{gmol}$ 1; IR $\left(\mathrm{KBr}, \mathrm{cm}^{-1}\right) v_{\max }: 3345$ (N-H stretching), 2941 (C-H stretching of aromatic ring), 2711 ($\mathrm{CH}_{2}$ stretching), $1635 \quad(\mathrm{C}=\mathrm{C}$ stretching of aromatic ring), 1305 ( $\mathrm{S}=\mathrm{O}$ stretching); ${ }^{1} \mathrm{H}-\mathrm{NMR}$ (300 MHz, CD3OD): $\delta / p p m ~ 7.18$ (dd, $J=7.5,2.4$ $\mathrm{Hz}, 2 \mathrm{H}, \mathrm{H}-2, \mathrm{H}-6), 7.11-6.97$ (m, 3H, H-3 to H-5), 6.95 (s, 2H, H-3', H-5'), 3.06 (t, J=7.2 Hz, 2H, $\mathrm{H}-8), 2.64$ (t, J = $7.5 \mathrm{~Hz}, 2 \mathrm{H}, \mathrm{H}-7), 2.63(\mathrm{~s}, 6 \mathrm{H}$, $\left.\mathrm{CH}_{3}-2^{\prime}, \mathrm{CH}_{3}-6^{\prime}\right), 2.26$ (s, 3H, $\left.\mathrm{CH}_{3}-4^{\prime}\right)$; $\operatorname{EIMS}(\mathrm{m} / \mathrm{z})$ : $303\left[\mathrm{M}^{+}, 183\left[\mathrm{C}_{9} \mathrm{H}_{11} \mathrm{O}_{2} \mathrm{~S}\right]^{+}, 119\left[\mathrm{C}_{9} \mathrm{H}_{11}\right]^{+}, 120\right.$ $\left[\mathrm{C}_{8} \mathrm{H}_{10} \mathrm{~N}\right]^{+}, 105\left[\mathrm{C}_{8} \mathrm{H}_{9}\right]^{+}, 91\left[\mathrm{C}_{7} \mathrm{H}_{7}\right]^{+}, 77\left[\mathrm{C}_{6} \mathrm{H}_{5}\right]^{+}, 65$ $\left[\mathrm{C}_{5} \mathrm{H}_{5}\right]^{+}, 51\left[\mathrm{C}_{4} \mathrm{H}_{3}\right]^{+}$.

\section{N-(2-Phenylethyl)-4-methoxybenzene sulfonamide (3d)}

Brown thick viscous liquid; Yield: 92.14\%; Mol. formula: $\mathrm{C}_{15} \mathrm{H}_{17} \mathrm{NO}_{3} \mathrm{~S}$; Mol. mass: $291 \mathrm{gmol}^{-1}$; IR $\left(\mathrm{KBr}, \mathrm{cm}^{-1}\right) v_{\text {max }}: 3332$ (N-H stretching), 2946 (C$\mathrm{H}$ stretching of aromatic ring), $2740 \quad\left(-\mathrm{CH}_{2}\right.$ stretching), 1620 ( $\mathrm{C}=\mathrm{C}$ stretching of aromatic ring), 1320 ( $\mathrm{S}=\mathrm{O}$ stretching); ${ }^{1} \mathrm{H}-\mathrm{NMR}(300 \mathrm{MHz}$, CD3OD): $\delta / p p m 7.72$ (d, J = 9.0 Hz, 2H, H-2', H6'), 7.21 (d, J = 7.5 Hz, H-2, H-6), 7.16 (t, $J=8.1$ $\mathrm{Hz}, 1 \mathrm{H}, \mathrm{H}-4), 7.11$ (dd, $J=8.1,1.5 \mathrm{~Hz}, 2 \mathrm{H}, 2 \mathrm{H}$, $\mathrm{H}-3, \mathrm{H}-5$ ), 7.04 (d, J=9.0 Hz, H-3', H-5'), 3.85 (s, $3 \mathrm{H}, \mathrm{CH}_{3} \mathrm{O}-4$ '), 3.03 (t, J=7.8 Hz, 2H, H-8), 2.70 $(\mathrm{t}, J=7.8 \mathrm{~Hz}, 2 \mathrm{H}, \mathrm{H}-7)$; EIMS $(\mathrm{m} / \mathrm{z}): 291[\mathrm{M}]^{+}$, $171\left[\mathrm{C}_{7} \mathrm{H}_{7} \mathrm{O}_{3} \mathrm{~S}\right]^{+}, 107\left[\mathrm{C}_{7} \mathrm{H}_{7} \mathrm{O}\right]^{+}, 120\left[\mathrm{C}_{8} \mathrm{H}_{10} \mathrm{~N}\right]^{+}$, $105\left[\mathrm{C}_{8} \mathrm{H}_{9}\right]^{+}, 91\left[\mathrm{C}_{7} \mathrm{H}_{7}\right]^{+}, 77\left[\mathrm{C}_{6} \mathrm{H}_{5}\right]^{+}, 65\left[\mathrm{C}_{5} \mathrm{H}_{5}\right]^{+}$, $51\left[\mathrm{C}_{4} \mathrm{H}_{3}\right]^{+}$.

\section{N-(2-Phenylethyl)-4-acetylbenzene sulfonamide (3e)}

White crystalline solid; Yield: 94.21\%; m.p.: 121 ${ }^{\circ} \mathrm{C}$; Mol. formula: $\mathrm{C}_{16} \mathrm{H}_{17} \mathrm{NO}_{3} \mathrm{~S}$; Mol. mass: 303 gmol $^{-1}$; IR $\left(\mathrm{KBr}, \mathrm{cm}^{-1}\right) v_{\text {max }}: 3341$ (N-H stretching), 2921 (C-H-stretching of aromatic ring), 2707 ($\mathrm{CH}_{2}$ stretching), $1605 \quad(\mathrm{C}=\mathrm{C}$ stretching of aromatic ring), 1321 ( $\mathrm{S}=\mathrm{O}$ stretching); ${ }^{1} \mathrm{H}-\mathrm{NMR}$

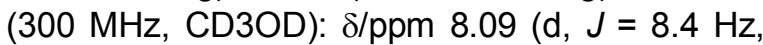
2H, H-2', H-6'), 8.02 (d, J = 8.7 Hz, H-3', H-5'), $7.63(\mathrm{~d}, \mathrm{~J}=8.4 \mathrm{~Hz}, 2 \mathrm{H}, \mathrm{H}-2, \mathrm{H}-6), 7.26-7.13$ (m, $3 \mathrm{H}, \mathrm{H}-3$ to H-5), 3.07 (t, J=7.2 Hz, H-8), 2.92 (t, $J=7.2 \mathrm{~Hz}, \mathrm{H}-7$ ), 1.50 (s, 3H, $\mathrm{CH}_{3} \mathrm{CO}-4$ '); EIMS $(\mathrm{m} / \mathrm{z}): 303[\mathrm{M}]^{+}, 183\left[\mathrm{C}_{8} \mathrm{H}_{7} \mathrm{SO}_{3}\right]^{+}, 120\left[\mathrm{C}_{8} \mathrm{H}_{10} \mathrm{~N}\right]^{+}$, $119\left[\mathrm{C}_{8} \mathrm{H}_{7} \mathrm{O}\right]^{+}, 105\left[\mathrm{C}_{8} \mathrm{H}_{9}\right]^{+}, 91\left[\mathrm{C}_{7} \mathrm{H}_{7}\right]^{+}, 77\left[\mathrm{C}_{6} \mathrm{H}_{5}\right]^{+}$, $65\left[\mathrm{C}_{5} \mathrm{H}_{5}\right]^{+}, 51\left[\mathrm{C}_{4} \mathrm{H}_{3}\right]^{+}$.
N-(2-Phenylethyl)-4-acetamidobenzene sulfonamide (3f)

White Crystalline Solid; Yield: 91.21\%; m.p: 107 ${ }^{\circ} \mathrm{C}$, Mol. formula: $\mathrm{C}_{16} \mathrm{H}_{18} \mathrm{~N}_{2} \mathrm{O}_{3} \mathrm{~S}$; Mol. mass: 318 gmol $^{-1}$; IR $\left(\mathrm{KBr}, \mathrm{cm}^{-1}\right) \quad v_{\text {max }}$ : $3301 \quad(\mathrm{~N}-\mathrm{H}$ stretching), 2921 (C-H stretching of aromatic ring), $2715 \quad\left(-\mathrm{CH}_{2}\right.$ stretching), $1615 \quad(\mathrm{C}=\mathrm{C}$ stretching of aromatic ring), $1311 \quad(\mathrm{~S}=\mathrm{O}$ stretching); ${ }^{1} \mathrm{H}-\mathrm{NMR}$ (300 MHz, CD3OD): $\delta / p p m$ $7.52\left(\mathrm{~d}, J=8.1 \mathrm{~Hz}, 2 \mathrm{H}, \mathrm{H}-3^{\prime}, \mathrm{H}-5^{\prime}\right), 7.32$ (d, $J=$ $\left.8.4 \mathrm{~Hz}, 2 \mathrm{H}, \mathrm{H}-2^{\prime}, \mathrm{H}-6^{\prime}\right), 7.23$ (d, J = 7.2 Hz, 2H, $\mathrm{H}-2, \mathrm{H}-6), 7.15$ (t, J = 6.9 Hz, 1H, H-4), 7.09 (t, J $=7.2 \mathrm{~Hz}, 2 \mathrm{H}, \mathrm{H}-7), 2.13$ (s, 3H, $\mathrm{CH}_{3} \mathrm{CONH}-4$ ); EIMS (m/z): $318[\mathrm{M}]^{+}, 198\left[\mathrm{C}_{8} \mathrm{H}_{8} \mathrm{SO}_{3} \mathrm{~N}\right]^{+}, 134$ $\left[\mathrm{C}_{8} \mathrm{H}_{8} \mathrm{ON}\right]^{+}, \quad 120\left[\mathrm{C}_{8} \mathrm{H}_{10} \mathrm{~N}\right]^{+}, \quad 105\left[\mathrm{C}_{8} \mathrm{H}_{9}\right]^{+}, \quad 91$ $\left[\mathrm{C}_{7} \mathrm{H}_{7}\right]^{+}, 77\left[\mathrm{C}_{6} \mathrm{H}_{5}\right]^{+}, 65\left[\mathrm{C}_{5} \mathrm{H}_{5}\right]^{+}, 51\left[\mathrm{C}_{4} \mathrm{H}_{3}\right]^{+}$.

\section{N-(2-Phenylethyl)-4-bromobenzene sulfonamide (3g)}

Yellowish white crystalline solid; Yield: 94.40\%; m.p.: $113{ }^{\circ} \mathrm{C}$; Mol. formula: $\mathrm{C}_{14} \mathrm{H}_{14} \mathrm{BrNO}_{2} \mathrm{~S}$; Mol. mass: $339 \mathrm{gmol}^{-1}$; IR $\left(\mathrm{KBr}, \mathrm{cm}^{-1}\right) v_{\text {max }}: 3317(\mathrm{~N}-\mathrm{H}$ stretching), 2925 (C-H stretching of aromatic ring), $2701 \quad\left(-\mathrm{CH}_{2}\right.$ stretching), $1619 \quad(\mathrm{C}=\mathrm{C}$ stretching of aromatic ring), $1301 \quad(\mathrm{~S}=\mathrm{O}$ stretching); ${ }^{1} \mathrm{H}-\mathrm{NMR}$ (300 MHz, MeOD): $\delta / \mathrm{ppm}$ $7.68\left(\mathrm{~d}, J=8.4 \mathrm{~Hz}, 2 \mathrm{H}, \mathrm{H}-3^{\prime}, \mathrm{H}-5^{\prime}\right), 7.65$ (d, $J=$ $\left.8.1 \mathrm{~Hz}, 2 \mathrm{H}, \mathrm{H}-2^{\prime}, \mathrm{H}-6^{\prime}\right), 7.21-7.11(\mathrm{~m}, 5 \mathrm{H}, \mathrm{H}-2$ to $\mathrm{H}-6), 3.08$ (t, $J=6.9 \mathrm{~Hz}, 2 \mathrm{H}, \mathrm{H}-8), 2.72$ (t, $J=6.9$ $\mathrm{Hz}, 2 \mathrm{H}, \mathrm{H}-7)$; EIMS (m/z): $341[\mathrm{M}+2]^{+}, 339$ [M] $^{+}$, $220\left[\mathrm{C}_{6} \mathrm{H}_{4} \mathrm{BrSO}_{2}\right]^{+}, 156\left[\mathrm{C}_{6} \mathrm{H}_{4} \mathrm{Br}\right]^{+}, 120\left[\mathrm{C}_{8} \mathrm{H}_{10} \mathrm{~N}\right]^{+}$, $105\left[\mathrm{C}_{8} \mathrm{H}_{9}\right]^{+}, 91\left[\mathrm{C}_{7} \mathrm{H}_{7}\right]^{+}, 77\left[\mathrm{C}_{6} \mathrm{H}_{5}\right]^{+}, 65\left[\mathrm{C}_{5} \mathrm{H}_{5}\right]^{+}$, $51\left[\mathrm{C}_{4} \mathrm{H}_{3}\right]^{+}$.

\section{N-(2-Phenylethyl)-4-chlorobenzene sulfonamide (3h)}

White crystalline solid; Yield: 94.25\%; m.p.: 101 ${ }^{\circ} \mathrm{C}$; Mol. formula: $\mathrm{C}_{14} \mathrm{H}_{14} \mathrm{CINO}_{2} \mathrm{~S}$; Mol. mass: 295 gmol $^{-1}$; IR $\left(\mathrm{KBr}, \mathrm{cm}^{-1}\right) v_{\text {max }}: 3327$ (N-H stretching), 2913 (C-H stretching of aromatic ring), 2714 ($\mathrm{CH}_{2}$ stretching), $1614 \quad(\mathrm{C}=\mathrm{C}$ stretching of aromatic ring), 1310 ( $\mathrm{S}=\mathrm{O}$ stretching); ${ }^{1} \mathrm{H}-\mathrm{NMR}$ (300 MHz, CD3OD): $\delta / p p m ~ 7.75$ (d, $J=8.7 \mathrm{~Hz}$, 2H, H-2', H-6'), 7.52 (d, J = 8.4 Hz, 2H, H-3', H$\left.5^{\prime}\right), 7.23$ (d, J = 7.2, 2H, H-2, H-6), 7.17 (t, J = 7.2 $\mathrm{Hz}, 1 \mathrm{H}, \mathrm{H}-4), 7.10$ (d, J = 7.2 Hz, 2H, H-3, H-5), 3.09 (t, J = 7.2 Hz, 2H, H-8), 2.72 (t, J=7.5 Hz, $2 \mathrm{H}, \mathrm{H}-7)$; $\operatorname{EIMS}(\mathrm{m} / \mathrm{z}): 297[\mathrm{M}+2]^{+}, 295[\mathrm{M}]^{+}, 175$ $\left[\mathrm{C}_{6} \mathrm{H}_{4} \mathrm{SO}_{2} \mathrm{Cl}\right]^{+}, 120\left[\mathrm{C}_{8} \mathrm{H}_{10} \mathrm{~N}\right]^{+}, 111\left[\mathrm{C}_{6} \mathrm{H}_{4} \mathrm{Cl}\right]^{+}, 105$ $\left[\mathrm{C}_{8} \mathrm{H}_{9}\right]^{+}, 91\left[\mathrm{C}_{7} \mathrm{H}_{7}\right]^{+}, 77\left[\mathrm{C}_{6} \mathrm{H}_{5}\right]^{+}, 65\left[\mathrm{C}_{5} \mathrm{H}_{5}\right]^{+}, 51$ $\left[\mathrm{C}_{4} \mathrm{H}_{3}\right]^{+}$.

\section{N-(2-Phenylethyl)-2,3-dichlorobenzene sulfonamide (3i)}

White crystalline solid; Yield: 92.31\%; m.p.: 115 ${ }^{\circ} \mathrm{C}$; Mol. formula: $\mathrm{C}_{14} \mathrm{H}_{13} \mathrm{Cl}_{2} \mathrm{NO}_{2} \mathrm{~S}$; Mol. mass: 329 $\mathrm{gmol}^{-1}$; IR $\left(\mathrm{KBr}, \mathrm{cm}^{-1}\right) v_{\text {max }}: 3331$ (N-H stretching), 2901 (C-H stretching of aromatic ring), 2705 ($\mathrm{CH}_{2}$ stretching), $1599 \quad(\mathrm{C}=\mathrm{C}$ stretching of aromatic ring), 1301 ( $\mathrm{S}=\mathrm{O}$ stretching); ${ }^{1} \mathrm{H}-\mathrm{NMR}$ 


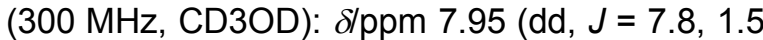
$\left.\mathrm{Hz}, 1 \mathrm{H}, \mathrm{H}-6^{\prime}\right), 7.71$ (dd, $J=8.1,1.5 \mathrm{~Hz}, 1 \mathrm{H}, \mathrm{H}-4^{\prime}$ ), 7.40 (t, $\left.J=8.1 \mathrm{~Hz}, 1 \mathrm{H}, \mathrm{H}-5^{\prime}\right), 7.40$ (t, $J=8.1 \mathrm{~Hz}$, $\left.1 \mathrm{H}, \mathrm{H}^{\prime} 5^{\prime}\right), 7.19$ (d, $\left.J=6.9 \mathrm{~Hz}, 2 \mathrm{H}, \mathrm{H}-2, \mathrm{H}-6\right), 7.12$ $(\mathrm{t}, J=6.9 \mathrm{~Hz}, 1 \mathrm{H}, \mathrm{H}-4), 7.06(\mathrm{dd}, J=7.8,1.2 \mathrm{~Hz}$, $2 \mathrm{H}, \mathrm{H}-3, \mathrm{H}-5), 3.19$ (t, J = 7.2 Hz, 2H, H-8), 2.70 (t, $J=7.2 \mathrm{~Hz}, 2 \mathrm{H}, \mathrm{H}-7)$; EIMS $(\mathrm{m} / \mathrm{z}): 333[\mathrm{M}+4]^{+}$, $331[\mathrm{M}+2]^{+}, 329[\mathrm{M}]^{+}, 209\left[\mathrm{C}_{6} \mathrm{H}_{3} \mathrm{O}_{2} \mathrm{Cl}_{2} \mathrm{~S}\right]^{+}, 145$ $\left[\mathrm{C}_{6} \mathrm{H}_{3} \mathrm{Cl}_{2}\right]^{+}, 120 \quad\left[\mathrm{C}_{8} \mathrm{H}_{10} \mathrm{~N}\right]^{+}, \quad 105 \quad\left[\mathrm{C}_{8} \mathrm{H}_{9}\right]^{+}, \quad 91$ $\left[\mathrm{C}_{7} \mathrm{H}_{7}\right]^{+}, 77\left[\mathrm{C}_{6} \mathrm{H}_{5}\right]^{+}, 65\left[\mathrm{C}_{5} \mathrm{H}_{5}\right]^{+}, 51\left[\mathrm{C}_{4} \mathrm{H}_{3}\right]^{+}$.

\section{N-(2-Phenylethyl)-2,5-dichlorobenzene sulfonamide (3j)}

White crystalline solid; Yield: 93.21\%; m.p.: 101 ${ }^{\circ} \mathrm{C}$, Mol. formula: $\mathrm{C}_{14} \mathrm{H}_{13} \mathrm{Cl}_{2} \mathrm{NO}_{2} \mathrm{~S}$; Mol. mass: 329 $\mathrm{gmol}^{-1}$; IR $\left(\mathrm{KBr}, \mathrm{cm}^{-1}\right) v_{\max }: 3319$ (N-H stretching), 2912 (C-H stretching of aromatic ring), 2719 ($\mathrm{CH}_{2}$ stretching), $1621 \quad(\mathrm{C}=\mathrm{C}$ stretching of aromatic ring), 1321 ( $\mathrm{S}=\mathrm{O}$ stretching), ${ }^{1} \mathrm{H}-\mathrm{NMR}$ (300 MHz, CD3OD): $\delta / \mathrm{ppm} 7.92(\mathrm{~d}, J=2.1 \mathrm{~Hz}$, $\left.1 \mathrm{H}, \mathrm{H}-6^{\prime}\right), 7.54\left(\mathrm{dd}, J=8.4,2.1 \mathrm{~Hz}, 1 \mathrm{H}, \mathrm{H}-4^{\prime}\right)$, 7.50 (d, J = 8.4 Hz, 1H, H-3'), 7.19 (d, J = 7.5 Hz, $2 \mathrm{H}, \mathrm{H}-2, \mathrm{H}-6), 7.12(\mathrm{t}, J=7.5 \mathrm{~Hz}, 1 \mathrm{H}, \mathrm{H}-4), 7.07$ $(\mathrm{dd}, J=8.1,1.2 \mathrm{~Hz}, 2 \mathrm{H}, \mathrm{H}-3, \mathrm{H}-5), 3.29$ (t, $J=$ $7.5 \mathrm{~Hz}, 2 \mathrm{H}, \mathrm{H}-8), 2.72(\mathrm{t}, J=7.2 \mathrm{~Hz}, \mathrm{H}-7$ ); EIMS $(\mathrm{m} / \mathrm{z}): 333[\mathrm{M}+4]^{+}, 331[\mathrm{M}+2]^{+}, 329[\mathrm{M}]^{+}, 209$ $\left[\mathrm{C}_{6} \mathrm{H}_{3} \mathrm{Cl}_{2} \mathrm{O}_{2} \mathrm{~S}\right]^{+}, 145\left[\mathrm{C}_{6} \mathrm{H}_{3} \mathrm{Cl}_{2}\right]^{+}, 120\left[\mathrm{C}_{8} \mathrm{H}_{10} \mathrm{~N}\right]^{+}$, $105\left[\mathrm{C}_{8} \mathrm{H}_{9}\right]^{+}, 91\left[\mathrm{C}_{7} \mathrm{H}_{7}\right]^{+}, 77\left[\mathrm{C}_{6} \mathrm{H}_{5}\right]^{+}, 65\left[\mathrm{C}_{5} \mathrm{H}_{5}\right]^{+}$, $51\left[\mathrm{C}_{4} \mathrm{H}_{3}\right]^{+}$.

\section{N-(2-Phenylethyl)-2,4-dinitrobenzene sulfonamide (3k)}

Yellowish white crystalline solid; Yield: 91.34\%; m.p.: $101{ }^{\circ} \mathrm{C}$; Mol. formula: $\mathrm{C}_{14} \mathrm{H}_{13} \mathrm{~N}_{3} \mathrm{O}_{6} \mathrm{~S}$; Mol. mass: $351 \mathrm{gmol}^{-1}$; IR $\left(\mathrm{KBr}, \mathrm{cm}^{-1}\right) v_{\max }: 3311(\mathrm{~N}-\mathrm{H}$ stretching), 2931 (C-H stretching of aromatic ring), $2725 \quad\left(-\mathrm{CH}_{2}\right.$ stretching $), \quad 1607 \quad(\mathrm{C}=\mathrm{C}$ stretching of aromatic ring), $1313 \quad(\mathrm{~S}=\mathrm{O}$ stretching); ${ }^{1} \mathrm{H}-\mathrm{NMR}$ (300 MHz, CD3OD): $\delta / \mathrm{ppm}$ $8.62\left(\mathrm{~d}, J=2.4 \mathrm{~Hz}, 1 \mathrm{H}, \mathrm{H}-3^{\prime}\right), 8.44$ (dd, $J=8.7$, $\left.2.1 \mathrm{~Hz}, \mathrm{H}-5^{\prime}\right), 8.09$ (d, $\left.J=8.7 \mathrm{~Hz}, 1 \mathrm{H}, \mathrm{H}-6^{\prime}\right), 7.15-$ $7.03(\mathrm{~m}, 5 \mathrm{H}, \mathrm{H}-2$ to $\mathrm{H}-6), 3.39(\mathrm{t}, J=7.2 \mathrm{~Hz}, 2 \mathrm{H}$, $\mathrm{H}-8), 2.78$ (t, $J=6.9 \mathrm{~Hz}, 2 \mathrm{H}, \mathrm{H}-7)$; $\operatorname{EIMS}(\mathrm{m} / \mathrm{z})$ :
$351[\mathrm{M}]^{+}, 231\left[\mathrm{C}_{6} \mathrm{H}_{3} \mathrm{O}_{6} \mathrm{~N}_{2} \mathrm{~S}\right]^{+}, 167\left[\mathrm{C}_{6} \mathrm{H}_{3} \mathrm{O}_{4} \mathrm{~N}_{2}\right]^{+}$, $120\left[\mathrm{C}_{8} \mathrm{H}_{10} \mathrm{~N}\right]^{+}, 105\left[\mathrm{C}_{8} \mathrm{H}_{9}\right]^{+}, \quad 91 \quad\left[\mathrm{C}_{7} \mathrm{H}_{7}\right]^{+}, 77$ $\left[\mathrm{C}_{6} \mathrm{H}_{5}\right]^{+}, 65\left[\mathrm{C}_{5} \mathrm{H}_{5}\right]^{+}, 51\left[\mathrm{C}_{4} \mathrm{H}_{3}\right]^{+}$.

\section{N-(2-Phenylethyl)naphthalen-2-ylsulfonamide (3I)}

Yellow crystalline solid; Yield: $94.51 \%$; m.p.: 92 ${ }^{\circ} \mathrm{C}$; Mol. formula: $\mathrm{C}_{18} \mathrm{H}_{17} \mathrm{NO}_{2} \mathrm{~S}$; Mol. mass: 311 $\mathrm{gmol}^{-1}$; IR $\left(\mathrm{KBr}, \mathrm{cm}^{-1}\right) v_{\text {max }}: 3340$ (N-H stretching), 2915 (C-H stretching of aromatic ring), 2731 ($\mathrm{CH}_{2}$ stretching), $1619 \quad(\mathrm{C}=\mathrm{C}$ stretching of aromatic ring), 1319 ( $\mathrm{S}=\mathrm{O}$ stretching); ${ }^{1} \mathrm{H}-\mathrm{NMR}$ (300 MHz, CD3OD): $\delta / p p m 8.37\left(\mathrm{~s}, 1 \mathrm{H}, \mathrm{H}-1^{\prime}\right)$, $8.17\left(\mathrm{t}, J=8.4 \mathrm{~Hz}, \mathrm{H}-6^{\prime}\right), 8.00(\mathrm{~d}, J=8.7 \mathrm{~Hz}, \mathrm{H}-$ 4'), 7.95 (d, $\left.J=9.3 \mathrm{~Hz}, \mathrm{H}-3^{\prime}\right), 7.78$ (dd, $J=8.7$, $\left.1.8 \mathrm{~Hz}, \mathrm{H}-5^{\prime}\right), 7.65\left(\mathrm{dd}, J=7.2,1.5 \mathrm{~Hz}, 1 \mathrm{H}, \mathrm{H}-8^{\prime}\right)$, $7.63\left(\mathrm{dd}, J=6.9,1.5 \mathrm{~Hz}, 1 \mathrm{H}, \mathrm{H}-7^{\prime}\right), 7.17$ (dd, $J=$ 7.2, $1.2 \mathrm{~Hz}, 2 \mathrm{H}, \mathrm{H}-2, \mathrm{H}-6), 7.11$ (t, $J=6.9 \mathrm{~Hz}$, $1 \mathrm{H}, \mathrm{H}-4), 7.06(\mathrm{~d}, J=6.9 \mathrm{~Hz}, 2 \mathrm{H}, \mathrm{H}-3, \mathrm{H}-5), 3.18$ (t, $J=7.2 \mathrm{~Hz}, 2 \mathrm{H}, \mathrm{H}-8), 2.71(\mathrm{t}, J=7.5 \mathrm{~Hz}, 2 \mathrm{H}$, $\mathrm{H}-7)$; $\operatorname{EIMS~(m/z):~} 311[\mathrm{M}]^{+}, 191\left[\mathrm{C}_{10} \mathrm{H}_{7} \mathrm{SO}_{2}\right]^{+}, 127$ $\left[\mathrm{C}_{10} \mathrm{H}_{7}\right]^{+}, 120\left[\mathrm{C}_{8} \mathrm{H}_{10} \mathrm{~N}\right]^{+}, 105\left[\mathrm{C}_{8} \mathrm{H}_{9}\right]^{+}, 91\left[\mathrm{C}_{7} \mathrm{H}_{7}\right]^{+}$, $77\left[\mathrm{C}_{6} \mathrm{H}_{5}\right]^{+}, 65\left[\mathrm{C}_{5} \mathrm{H}_{5}\right]^{+}, 51\left[\mathrm{C}_{4} \mathrm{H}_{3}\right]^{+}$.

\section{Enzyme inhibition activity}

A series of sulfonamides derived from 1-amino-2phenylethane was synthesized by the protocol sketched in scheme 1 and evaluated for antienzymatic activity by screening against acetyl cholinesterase (AChE), butyryl cholinesterase (BChE) and lipoxygenase (LOX) enzymes. The enzyme inhibition activity of the sulfonamide prepared by the reaction of 2-phenylethylamine and benzenesulfonyl chloride and its derivatives has previously been evaluated by our group [15]. Here, we further prepared sulfonyl derivatives of 1-amino-2-phenylethane (2-phenylethylamine) to evaluate their biological activities in search of new suitable molecules. The results indicate that these molecules are suitable inhibitors of both cholinesterase enzymes but moderately active against lipoxygenase enzyme.

Table 1: Enzyme inhibition activities of synthesized compounds (3a-I)

\begin{tabular}{|c|c|c|c|c|c|c|}
\hline \multirow[b]{2}{*}{ Compd. } & \multicolumn{2}{|c|}{ AChE } & \multicolumn{2}{|c|}{ BChE } & \multicolumn{2}{|c|}{ LOX } \\
\hline & Inhibition (\%) & $\mathrm{IC}_{50}(\mu \mathrm{M})$ & Inhibition (\%) & $\mathrm{IC}_{50}(\mu \mathrm{M})$ & $\begin{array}{l}\text { Inhibition } \\
\text { (\%) }\end{array}$ & $\mathrm{IC}_{50}(\mu \mathrm{M})$ \\
\hline $3 \mathbf{a}$ & $82.34 \pm 3.76$ & $402.65 \pm 1.59$ & $69.04 \pm 2.98$ & $304.14 \pm 1.95$ & $81.45 \pm 0.31$ & $251.07 \pm 0.65$ \\
\hline $3 b$ & $77.94 \pm 2.90$ & $324.35 \pm 1.23$ & $64.25 \pm 3.87$ & $229.26 \pm 1.72$ & $27.63 \pm 0.67$ & - \\
\hline $3 c$ & $86.92 \pm 2.51$ & $82.93 \pm 0.15$ & $66.65 \pm 2.92$ & $143.15 \pm 0.81$ & $33.90 \pm 0.54$ & - \\
\hline $3 d$ & $2.95 \pm 3.82$ & - & $86.71 \pm 2.25$ & $45.65 \pm 0.48$ & $38.81 \pm 0.27$ & - \\
\hline $3 e$ & $82.25 \pm 3.96$ & $184.15 \pm 0.62$ & $79.02 \pm 3.24$ & $212.65 \pm 1.85$ & $66.27 \pm 0.51$ & $342.76 \pm 0.78$ \\
\hline $3 f$ & $23.67 \pm 3.69$ & - & $13.01 \pm 3.67$ & - & $16.23 \pm 0.19$ & - \\
\hline $3 \mathrm{~g}$ & $45.52 \pm 3.98$ & $>500$ & $70.71 \pm 2.45$ & $294.13 \pm 1.63$ & $60.90 \pm 0.34$ & $356.87 \pm 0.59$ \\
\hline $3 h$ & $55.14 \pm 3.22$ & $382.21 \pm 1.67$ & $71.16 \pm 2.73$ & $233.24 \pm 1.83$ & $64.36 \pm 0.27$ & $347.14 \pm 0.97$ \\
\hline $3 i$ & $92.23 \pm 2.31$ & $362.55 \pm 1.94$ & $78.96 \pm 3.51$ & $254.75 \pm 1.63$ & $22.31 \pm 0.17$ & - \\
\hline $3 j$ & $85.16 \pm 2.52$ & $326.41 \pm 1.43$ & $81.75 \pm 2.35$ & $234.85 \pm 1.69$ & $5.45 \pm 0.77$ & - \\
\hline $3 k$ & $56.53 \pm 3.25$ & $421.12 \pm 1.78$ & $84.67 \pm 3.50$ & $129.57 \pm 0.75$ & $59.27 \pm 0.41$ & $364.43 \pm 0.91$ \\
\hline 31 & $88.52 \pm 2.76$ & $185.15 \pm 0.56$ & $80.76 \pm 3.79$ & $162.39 \pm 0.94$ & $69.60 \pm 0.61$ & $330.43 \pm 0.85$ \\
\hline Control & $91.29 \pm 1.17^{\mathrm{a}}$ & $0.04 \pm 0.0001^{a}$ & $82.82 \pm 1.09^{\mathrm{a}}$ & $0.85 \pm 0.0001^{\mathrm{a}}$ & $93.79 \pm 1.27^{b}$ & $22.4 \pm 1.3^{\mathrm{b}}$ \\
\hline
\end{tabular}




\section{DISCUSSION}

Compound $3 \mathbf{a}$ was obtained as white crystalline solid having yield of $92.72 \%$ and m.p. of $98{ }^{\circ} \mathrm{C}$. IR spectrum supported the presence of sulfamoyl group by stretching of $\mathrm{N}-\mathrm{H}$ bond at $3310 \mathrm{~cm}^{-1}$ and that of $\mathrm{S}=\mathrm{O}$ group at $1320 \mathrm{~cm}^{-1}$. Molecular formula was also established by EI-MS molecular ion peak at $\mathrm{m} / \mathrm{z} 275$ and also by counting the number of protons in ${ }^{1} \mathrm{H}-\mathrm{NMR}$ spectrum. EI-MS gave two prominent fragment peaks at $\mathrm{m} / \mathrm{z} 155$ for toluene sulfonyl cation and at $\mathrm{m} / \mathrm{z} 120$ for the cation of phenylethyl amino group.

In the ${ }^{1} \mathrm{H}-\mathrm{NMR}$ spectrum, two doublets appeared at $\delta 7.68\left(\mathrm{~d}, J=8.1 \mathrm{~Hz}, 2 \mathrm{H}, \mathrm{H}-2^{\prime}, \mathrm{H}-66^{\prime}\right)$ for two protons in the vicinity of strong electron withdrawing sulfonyl group and $7.33(\mathrm{~d}, J=8.1$ $\left.\mathrm{Hz}, 2 \mathrm{H}, \mathrm{H}-3^{\prime}, \mathrm{H}-5^{\prime}\right)$ for the other two protons of $p$ disubstituted benzene ring. The three signals appearing at $\delta 7.23(\mathrm{dd}, J=7.5,1.5 \mathrm{~Hz}, 2 \mathrm{H}, \mathrm{H}-2$, $\mathrm{H}-6), 7.15$ (t, $J=7.8 \mathrm{~Hz}, 1 \mathrm{H}, \mathrm{H}-4)$ and 7.09 (dd, $J$ $=7.8,1.2 \mathrm{~Hz}, 2 \mathrm{H}, \mathrm{H}-3, \mathrm{H}-5$ ) were assigned for five proton substituted at ortho, para and meta position in the benzene ring. The signals appearing in aliphatic region at $\delta 3.03(\mathrm{t}, \mathrm{J}=7.8$ $\mathrm{Hz}, 2 \mathrm{H}, \mathrm{H}-8)$ and $2.69(\mathrm{t}, \mathrm{J}=7.2 \mathrm{~Hz}, 2 \mathrm{H}, \mathrm{H}-7)$ confirmed the presence of two adjacent methylene groups in the molecule; and at $\delta 2.40$ (s, $\left.3 \mathrm{H}, \mathrm{CH}_{3}-4\right)$ supported the presence of methyl group attached at para position of benzene ring linked with sulfonyl group. On the basis of these evidences, the structure of $3 \mathbf{a}$ was named as $N$ (2-phenylethyl)-4-methylbenzenesulfonamide.

Likewise the structures of other synthesized compounds (3b-I) were corroborated by ${ }^{1} \mathrm{H}$ NMR, IR and mass spectra data.

The screening of the synthesized molecules against acetyl cholinesterase (AChE) revealed that the most of the molecules exhibited inhibition potential except $\mathbf{3 d}, \mathbf{3 f}$ and $\mathbf{3 g}$ as shown by their $\mathrm{IC}_{50}$ values. Among these molecules, $\mathrm{N}-(2-$ phenylethyl)-2, 4, 6-trimethylbenzenesulfonamide (3c) was highly active. This molecule showed the inhibition potential probably because of the presence of trimethyl substituted benzene ring which exhibited more interaction with the active site of the enzyme to block it. The order of inhibition potential of all the molecules was found to be as, $3 c>3 e>3 l>3 b>3 j>3 i>3 h>3 a>$ 3k.

Butyryl cholinesterase enzyme was inhibited by almost all the molecules with higher $I_{50}$ values relatively but still $\mathbf{3 f}$ was inactive. The most active molecule was $N$-(2-phenylethyl)-4-methoxy benzenesulfonamide (3d) and the most credibly due to $p$-substituted methoxy benzyl group which exhibited $\mathrm{H}$-bonding and also $\pi-\pi$ interactions with amino acid residues associated with the active site of this enzyme.The activity of the molecules was in the following rank order: $3 \mathbf{d}>$ $3 k>3 c>3 l>3 e>3 b>3 h>3 j>3 i>3 g>3 a$.

The synthesized compounds showed moderate activities against lipoxygenase enzyme. The high $I_{50}$ values of the active molecules against this enzyme indicate that they were less active. The rank order of inhibition of the molecules was $3 a>$ $3 \mathrm{l}>\mathbf{3 e}>\mathbf{3 h}>\mathbf{3 g}>\mathbf{3 k}$. Half of the molecules of the synthesized series were inactive.

\section{CONCLUSION}

The series of synthesized sulfonamides can be obtained in yield by a facile and benign method using water as reaction medium. Compound $\mathbf{3 f}$ remained inactive against all the three enzymes taken into account. Overall, the compounds are active against both cholinesterase enzymes but less potent against lipoxygenase enzyme. These findings may be helpful in the efforts to design and search for new drug candidates for Alzheimer's disease.

\section{REFERENCES}

1. Sarmah AK, Meyer MT, Boxall ABA. A global perspective on the use, sales, exposure pathways, occurrence, fate and effects of veterinary antibiotics (VAs) in the environment. Chemsphere 2006; 65(5): 725-759.

2. Remko M, Lieth CWV. Theoretical study of gas phase acidity, pka, lipophilicity and solubility of some biologically active sulfonamides. Bioorg Med Chem 2004; 12(20): 5395-5403.

3. Boyd AE. Sulfonyl urea receptors ion, channels and fruit flies. Diabetes 1988; 237: 847-850.

4. De Clercq E. New developments in anti-HIV chemotherapy. Curr Med Chem 2001; 8: 15431572.

5. Jerry S, Riviere J. Sulfonamides veterinary pharmacology and therapeutics, edn 8, Ed. Richard Towa State, University Press 2001.

6. El-Sayed NS, El-Bendary RE, El-Ashry SM, El-Kerdawy MM. Synthesis and antitumour activity of new sulfonamide derivatives of thiadiazole [3,2a] pyrimidines. Eur J Med Chem 2011; 46(9): 37143720.

7. Tougu V. Acetylcholinesterase: Mechanism of catalysis and inhibition. Curr Med Chem 2001; 1: 155-170.

8. Gauthier S. Cholinergic adverse effects of cholinesterase inhibitors in Alzheimer's disease. Drug Aging 2001; 18: 853-862.

9. Abbasi MA, Aziz-ur-Rehman, Muhmood T, Khan KM, Ashraf M, Ejaz SA, Arshad S. Synthesis structural characterization and biological screening of various sulfa drugs derived from 2-anisidine. J Chem Soc Pak 2013; 35(2): 404-410.

10. Aziz-ur-Rehman, Rasool S, Abbasi MA, Khan KM, Ashraf M, Afzal I. Synthesis, characterization and biological screening of some 4-O-substituted derivatives of $\mathrm{N}$-(4-hydroxyphenyl)-N-methyl-4methylbenzenesulfonamide. Asian $J$ Pharm Bio Res 2012; 2(2): 100-105.

11. Aziz-ur-Rehman, Rasool $S$, Abbasi MA, Fatima A, Nafeesa K, Ahmad I, Afzal S. Synthesis, spectral analysis and biological screening of some new $N$ - 
(un)substituted N-(5-chloro-2-methoxyphenyl)-aryl sulfonamides. J Pharmacy Res 2013; 6: 559-564.

12. Aziz-ur-Rehman, Fatima $A$, Abbasi MA, Khan $K M$ Ashraf M, Ahmad I, Ejaz SA. Synthesis, characterization and biological screening of $N$ substituted-(5-chloro-2-

methoxyphenyl)benzenesulfonamide. Asian $\mathrm{J}$ Chem 2013; 25(7): 3735-3740.

13. Ellman GL, Courtney KD, Andres V, Featherstone RM. A new and rapid calorimetric determination of acetylcholinesterase activity. Biochem Pharmacol
1961; 7: 88-90. Baylac S, Racine P. Inhibition of 5lipoxygenase by essential oils and other natural fragrant extracts. Int J Aromather 2003; 13: 138142.

14. Aziz-ur-Rehman, Afroz S, Abbasi MA, Tanveer W, Khan KM, Ashraf M, Afzal I, Ambreen N. Synthesis characterization and biological screening of sulfonamides derived from 2-phenylethylamine. Pak J Pharm Sci 2012; 25(4): 809-814. 